\title{
Factors associated with favourable outcome in large hemispheric infarctions
}

\author{
Jie Li ${ }^{1}$, Ping Zhang ${ }^{1}$, Simiao $\mathrm{Wu}^{2}$, Xingyang $\mathrm{Yi}^{1}$, Chun Wang ${ }^{1^{*}}$ and Ming $\mathrm{Liu}^{2^{*}}$
}

\begin{abstract}
Background: Large hemispheric infarction $(L H I)$ is a devastating condition with high mortality and poor functional outcome in most conservatively treated patients. The purpose of this study was to explore factors associated with favorable outcome in patients with $\mathrm{LHI}$.

Methods: We prospectively enrolled consecutive patients with LHI. Favorable outcome was defined as a modified Rankin Scale (mRS) score of 0 to 3 at 90 days. Multivariate logistic regression analysis was employed to identify the independent factors associated with favorable outcome.

Results: Two hundred fifty-six cases with LHI were identified: 41 (16.0\%) died during hospitalization, 94 (36.7\%) died at 3 month, and 113 (44.1\%) survived with favorable outcome at day 90. Compared with patients with unfavorable outcome, the favorable cases were younger ( $55.8 \pm 14.7$ vs. $66.2 \pm 14.1$ ), had less history of hypertension (38.9\% vs. 59.3\%), lower baseline NIHSS score (median NIHSS score 11 vs. 17), lower blood pressure on admission (systolic $134.7 \pm 24.9$ vs. $145.1 \pm 26$. $1 \mathrm{mmHg}$; diastolic $80.2 \pm 14.9$ vs. $86.9 \pm 16.2 \mathrm{mmHg}$; respectively), lower level of baseline serum glucose (7.2 $\pm 3.3 \mathrm{vs.} 8.2 \pm 3$. $3 \mathrm{mmol} / \mathrm{L}$ ), a lower frequency of stroke-related complications (55.8\% vs. $91.4 \%)$, more use of antiplatelets (93.8\% vs. 57.1\%) and statins (46.9\% vs. $25.7 \%)$ in the acute phase of stroke, but less use of osmotic agents (69.9\% vs. 89.3\%), mechanical ventilation (1.8\% vs. $20.0 \%$ ) or decompressive hemicraniectomy (1.8\% vs. $15.7 \%$ ). Multivariable analysis identified the following factors associated with favorable outcome: age (odds ratio, OR 0.95, 95\% confidence interval [CI] 0.92-0.98, $p<0$. 001), baseline NIHSS score (OR 0.90, 95\% Cl 0.84-0.96, $p=0.002)$, statins used in acute phase (OR 2.49, 95\% Cl 1.10-5.65, $p=$ 0.029 ), brain edema (OR 0.05, 95\% Cl 0.01-0.21, $p<0.001$ ) and pneumonia (OR 0.42, 95\% Cl 0.19-0.93, $p=0.032$ ).
\end{abstract}

Conclusion: More than one third of patients with LHI have relatively favorable clinical outcomes at 90 days. Younger age, lower baseline NIHSS score, absence of brain edema and pneumonia, and statins used in the acute phase were associated with favorable outcome of patients with LHI at 90 days.

Keywords: Large hemispheric infarction, Brain edema, Decompressive hemicraniectomy, Complication, Favorable outcome

\section{Background}

Large hemispheric infarction (LHI), which usually refers to total or partial anterior circulation infarct caused by occlusion of internal carotid artery or the proximal middle cerebral artery (MCA), constitutes up to $10 \%$ of all supratentorial ischemic strokes [1]. Treatment of LHI has been a major unsolved problem in neurocritical care [2-4], with a high mortality rate of approximately $80 \%$ in conservatively treated patients [5].

\footnotetext{
*Correspondence: dysrmyysjnkwc@163.com; wyplmh@hotmail.com 'Department of Neurology, People's Hospital of Deyang City, No.173, North Taishan Road, Deyang 618000, Sichuan Province, People's Republic of China ${ }^{2}$ Stroke Clinical Research Unit, Department of Neurology, West China Hospital, Sichuan University, No. 37, GuoXue Xiang, Chengdu 610041, Sichuan Province, People's Republic of China
}

Although decompressive hemicraniectomy (DHC) within $48 \mathrm{~h}$ after symptom onset has been proven to benefit highly selected patients with LHI [6], only $0.3 \%$ of all ischemic stroke patients would be eligible for DHC on the basis of the strict eligibility criteria in the European hemicraniectomy trials [7]. Until recently, there is a paucity of high quality evidence for doctors and family members to make decisions on treatment for patients with LHI.

The principal cause of death and poor functional outcome in patients with LHI is post-ischemic brain swelling, with subsequent raised intracranial pressure, neurological deterioration, midline shift and brain herniation [8]. Thus, prediction of malignant brain edema using clinical and

(C) The Author(s). 2018 Open Access This article is distributed under the terms of the Creative Commons Attribution 4.0 International License (http://creativecommons.org/licenses/by/4.0/), which permits unrestricted use, distribution, and 
radiological variables has been thoroughly investigated for the last two decades [9-13]. It is noted that not all patients with LHI would develop the malignant MCA infarction ( $\mathrm{mMCAi}$ ) that is characterized by severe edema and mass effect [14]. On the other hand, post- ischemic brain edema may produce a series of clinical manifestations from being clinically silent to rapid neurological deterioration or even death, depending on stroke location, infarct size, age of patient, and degree of premorbid atrophy [15]. Current available data for LHI in general emphasize the prediction and management of maligant brain edema, but offer limited discussion of the factors associated with favourable outcome in patients with LHI specifically. Therefore, we conducted a prospective cohort study that aimed to elucidate the factors that correlate with favorable outcome in patients with LHI.

\section{Methods}

\section{Study design and subjects}

Between Oct 1, 2011 and Sep 30, 2014, patients with either a first-ever stroke or recurrent stroke were registered consecutively after they were admitted to the Department of Neurology, People's Hospital of Deyang City. Data were recorded at the time of assessment using a standardized structured form. Detailed methods for data collection have been previously described [16]. In present study, we enrolled patients who were admitted within 30 days from symptoms onset and diagnosed with LHI, which was defined as an ischemic stroke involving more than $50 \%$ of the territory of the MCA in computed tomography (CT) scan and/or standard magnetic resonance imaging (MRI), with or without involvement of the adjacent territories [17]. All patients had a brain CT scan before initial treatment. A second CT scan or MRI was performed within first 7 days of hospitalization. Other CT scans were performed if patients were suffered neurological deterioration, to identify brain edema or hemorrhagic transformation. We excluded cases with incomplete hospital records or missing imaging that would prevent complete data collection. We also excluded cases with preexisting score of more than 2 on the modified Rankin scale (mRS, a scale of 0 to 6 , with 0 indicating no symptoms and 6 indicating death) and lived dependently [18].

\section{Data collection and outcome}

Baseline data on age, sex, living environment (rural or urban), admission delay, initial stroke severity assessed by the National Institutes of Health Stroke Scale (NIHSS) score, baseline systolic and diastolic blood pressure, serum glucose on admission, vascular risk factors were collected. Vascular risk factors investigated in this study included hypertension, diabetes mellitus, dyslipidemia, coronary heart disease, atrial fibrillation, rheumatic heart disease, previous stroke/transient ischemic attack (TIA), current smoking and alcohol consumption, which have been described in a previous study [16]. The potential etiology of LHI was classified as large-artery atherosclerosis, cardio-embolism, stroke of other determined etiology and stroke of undetermined etiology according to the Trial of Org 10,172 in Acute Stroke Treatment (TOAST) criteria [19]. In-hospital treatment analyzed in our study included thrombolysis, DHC, mechanical ventilation, osmotic agents (such as mannitol), antiplatelet agents, anticoagulants, antihypertensives, statins and antidiabetics. DHC was conducted according the eligibility and exclusion criteria from the pooled analysis of three European randomized trials, when written informed consent was obtained from the patient or a legal representative [6]. Statins were used in patients whose stroke was presumed to be of atherosclerotic origin [20], or in patients regularly pretreated with statins [21], without consideration of baseline NIHSS score. Statins use was classified into two phases: previous stroke treatment or in the acute phase. Previous stroke treatment of statins referred to regular use of statins at least 1 month before stroke, and statins treatment in the acute phase was defined as statins initiated within $24 \mathrm{~h}$ after stroke onset and continuing for at least 3 days. The dosage was $\geq 20 \mathrm{mg}$ per day for atorvastatin, $\geq 40 \mathrm{mg}$ per day for simvastatin, or $\geq 10 \mathrm{mg}$ per day for rosuvastatin. Stroke-related complications, including both neurological and medical complications during hospitalization, were reviewed by our staff members from hospital records after patient discharge. Neurological complications included brain edema, hemorrhagic transformation, seizures/epilepsy, central hyperthermia and recurrent stroke, while medical complications during hospitalization included pneumonia, urinary tract infection, gastrointestinal bleeding, electrolyte disturbance, urinary incontinence, acute renal failure, deep venous thrombosis, bedsore and falls [22].

Patients were followed up at 90 days after stroke onset by using questionnaires via telephone interview or letter inquiries. The Primary outcomes measures in our study were 3-month favorable outcome (defined as a mRS score of 0 to $3[6])$.

\section{Statistical analyses}

Baseline characteristics were compared between favorable and unfavorable outcomes using student's t-tests, Mann-Whitney U tests, $X^{2}$ tests or Fisher exact tests, as appropriate.Univariate analysis was performed to test variables which may affect outcome. The included variables were: (1) age group, (2) NIHSS score on admission, (3) all the risk factors surveyed in our study, (4) in-hospital treatments and (5) stroke related complication. The odds ratios (ORs) for variables associated with favorable outcomes were determined using multivariable logistic regression analyses by the forced entry method adjusted for variables with $p<0.05$ on univariate analyses. All statistical levels quoted are 2-tailed and a value of $p<0.05$ was considered 
significant for all results. The $95 \%$ confidence intervals (CI) were calculated to describe the precision of the estimates. All statistical analysis was performed with SPSS v21.0 (SPSS, Chicago, IL).

\section{Results}

During the 3-year study period, 1542 patients with acute ischemic stroke were consecutively and prospectively registered. Of those, 256 (16.6\%) patients with LHI were enrolled in the present study [mean age: $61.6 \pm 15.3$ years; $133(52.0 \%)$ female; median NIHSS score on admission: 14]. Of these patients, $24(9.4 \%)$ received DHC and 30 (11.7\%) received mechanical ventilation. None of the patients received intra-arterial revascularization procedures. All LHI patients received cranial CT examination at least one time and 161 (62.9\%) patients received MRI. The median length of stay in hospital (LOS) was 9.5 days (range, 1 to 78$)$. 41 (16.0\%) patients died during hospitalization. At 90 days, 3 (1.2\%) patients was lost to follow-up. Among the entire cohort, 113 (44.1\%) patients had favorable outcomes and 94 (36.7\%) patients died at 90 days.

The baseline characteristics were compared between LHI patients with and without favorable outcomes (Table 1). Patients with favorable outcomes were younger $(55.8 \pm 14.7$ vs. $66.2 \pm 14.1, p<0.001$ ), had less history of hypertension (38.9\% vs. $59.3 \%, p=0.002)$, lower blood pressure on admission (systolic $134.7 \pm 24.9$ vs. $145.1 \pm 26.1 \mathrm{mmHg}, p=$ 0.001 ; diastolic $80.2 \pm 14.9$ vs. $86.9 \pm 16.2 \mathrm{mmHg}, p=0.001$; respectively), lower baseline serum glucose ( $7.2 \pm 3.3$ vs. 8.2 $\pm 3.3 \mathrm{mmol} / \mathrm{L}, p=0.015$ ), and a longer onset to admission time ( 26 vs. $24 \mathrm{~h}, p=0.014$ ) than those with unfavorable outcomes. The median total NIHSS score on admission was 11 in patients with favorable outcomes and 17 in those with unfavorable outcomes $(p<0.001)$. When stroke etiology of LHI is concerned, no significant difference was found between patients with and without favorable outcome $(p=0.222)$. The most common stroke subtype in both group was cardio-embolism $(42.5 \%$ and $53.6 \%$, respectively). 13 (11.5\%) patients with favorable outcome and 13 (9.3\%) without favorable outcome used statins previous to stroke. However, statins pretreatment did not have a significant association with favorable outcome in LHI in univariate analysis $(p=0.678)$. For in-hospital management of LHI, patients with favorable outcome more frequently used antiplatelets $(93.8 \%$ vs. $57.1 \%, p<0.001)$ and statins $(46.9 \%$ vs. $25.7 \%, p<0.001)$ in the acute phase of stroke, and less frequently used osmotic agents $(69.9 \%$ vs. $89.3 \%, p<0.001)$, mechanical ventilation $(1.8 \%$ vs. $20.0 \%, p<0.001)$, and DHC $(1.8 \%$ vs. $15.7 \%, p<0.001)$ (Table 2$)$.

Patients with favorable outcome had less stroke-related complications (55.8\% vs. $91.4 \%, p<0.001$; Table 3$)$. Compared with patients with unfavourable outcomes, those with favorable outcomes had a significantly lower rate of malignant brain edema $(4.4 \%$ vs. $53.6 \%, p<0.001)$, pneumonia (36.3\% vs. $67.1 \%, p<0.001)$, gastrointestinal bleeding (3.5\% vs. $17.9 \%, p=0.001)$, electrolyte disturbance $(19.5 \%$ vs. $40.0 \%, p<0.001)$, acute renal failure ( 0 vs. $12.9 \%, p<0.001)$, urinary incontinence $(7.1$ vs. $27.9 \%, p<$ $0.001)$ and bedsore (1.8 vs. $7.9 \%, p=0.042)$. However, there was no significant difference in the events rate of hemorrhagic transformation, seizures/epilepsy, central hyperthermia and recurrent stroke between patients with and without favorable outcomes (all $p>0.05$ ).

Variables that had potential confounding effects on the 3-month favorable outcome in univariate analysis $(p<0.05)$ and the final results of multivariate logistic regression are shown in Table 4 with OR and 95\% CI. After adjusting for potential confounding factors excluding stroke-related complications (see model 1), age (OR 0.95, 95\% CI 0.92-0.97), baseline NIHSS score (OR $0.87,95 \%$ CI $0.82-0.92$ ), history of hypertension (OR 0.38; 95\% CI 0.18-0.78), DHC (OR $0.11 ; 95 \%$ CI $0.01-0.92)$, statins use in acute phase (OR 2.75, 95\% CI 1.35-5.59) were independently associated with 3-month favorable outcome in LHI patients(all $P<0.05)$. When stroke-related complications were included into the multivariate logistic regression (see model 2), history of hypertension and DHC were no longer independently associated with 3-month favorable outcome, however, brain edema (OR 0.05, 95\% CI 0.01-0.21) and pneumonia (OR 0.42 , 95\% CI $0.19-0.93$ ) were independently associated with 3-month favorable outcome in LHI patients in addition to age (OR 0.95, 95\% CI $0.92-$ 0.98), baseline NIHSS score (OR 0.90, 95\% CI $0.84-$ $0.96)$ and statins used in acute phase(OR 2.49, 95\% CI 1.10-5.65) (all $P<0.05$ ).

\section{Discussion}

It has been reported that advanced age, initial stroke severity (NIHSS or Glasgow coma scale score), involvement of the adjacent (anterior or posterior cerebral artery) territories, anisocoria, early neurological deterioration, coronary artery disease, and internal carotid artery occlusion were the predictors of mortality and poor functional outcome in patients with LHI. However, all of these studies mentioned above were conducted in DHC cohorts [17, 23-29]. A number of clinical and radiological predictors of malignant brain edema, such as younger age, female sex, no previous ischemic stroke, history of hypertension, dominant hemisphere, have been extensively investigated for the last two decades [30]. A meta analysis included 23 studies and found that large infarct size especially involving more than $50 \%$ of the MCA territory and a perfusion deficit of more than $66 \%$ on CT scan to be the most reliable predictors of maligant edema formation [13]. Nevertheless, not all patients with LHI would develop 
Table 1 Baseline characteristics of LHI patients with favorable and unfavorable outcome

\begin{tabular}{|c|c|c|c|}
\hline & Unfavorable (mRS score 4-6) $(n=140)$ & Favorable (mRS score $0-3)(n=113)$ & $P$ value \\
\hline \multicolumn{4}{|l|}{ Age(years) } \\
\hline Mean \pm SD & $66.2 \pm 14.1$ & $55.8 \pm 14.7$ & $<0.001^{*}$ \\
\hline Median(range) & $69(17-99)$ & $57(15-88)$ & $<0.001+$ \\
\hline Female, n(\%) & $76(54.3)$ & $56(49.6)$ & $0.527 \neq$ \\
\hline Rural population, $\mathrm{n}(\%)$ & $43(30.7)$ & $41(36.3)$ & $0.421 \neq$ \\
\hline Time from onset(hours), median(range) & $24(1-720)$ & $26(3-720)$ & $0.014+$ \\
\hline NIHSS score on admission, median(range) & $17(5-33)$ & $11(4-31)$ & $<0.001+$ \\
\hline SBP on admission (mm Hg) & $145.1 \pm 26.1$ & $134.7 \pm 24.9$ & $0.001^{*}$ \\
\hline DBP on admission (mm Hg) & $86.9 \pm 16.2$ & $80.2 \pm 14.9$ & $0.001^{*}$ \\
\hline Serum glucose on admission(mmol/L) & $8.2 \pm 3.3$ & $7.2 \pm 3.3$ & $0.015^{*}$ \\
\hline \multicolumn{4}{|l|}{ Risk factors, n(\%) } \\
\hline Hypertension & $83(59.3)$ & $44(38.9)$ & $0.002 \neq$ \\
\hline Diabetes mellitus & $32(22.9)$ & $20(17.7)$ & $0.350 \neq$ \\
\hline Dyslipidemia & $22(15.7)$ & $25(22.1)$ & $0.198 \neq$ \\
\hline Coronary heart disease & $21(15.0)$ & $9(8.0)$ & $0.117 \neq$ \\
\hline Atrial fibrillation & $69(49.3)$ & $42(37.2)$ & $0.057 \neq$ \\
\hline Rheumatic heart disease & $27(19.3)$ & $31(27.4)$ & $0.135 \neq$ \\
\hline Current smoking & $30(21.4)$ & $27(23.9)$ & $0.653 \neq$ \\
\hline Alcohol consumption & $23(16.4)$ & 18(15.9) & $0.915 \neq$ \\
\hline Previous all strokes/TIA & $27(19.3)$ & 15(13.3) & $0.236 \neq$ \\
\hline Stroke in dominant hemisphere, $\mathrm{n}(\%)$ & $65(46.4)$ & $60(53.1)$ & $0.313 \neq$ \\
\hline TOAST classification, n(\%) & & & $0.222 \neq$ \\
\hline Large-artery atherosclerosis & $27(19.3)$ & $30(26.6)$ & \\
\hline Cardioembolism & 75(53.6) & $48(42.5)$ & \\
\hline Other determined etiology & $9(6.4)$ & $5(4.4)$ & \\
\hline Undetermined etiology & $29(20.7)$ & $30(26.6)$ & \\
\hline Statins pretreatment & $13(9.3)$ & 13(11.5) & $0.678 \neq$ \\
\hline
\end{tabular}

Abbreviations: SBP systolic blood pressure, DBP diastolic blood pressure, NIHSS National Institutes of Health Stroke Scale, GCS Glasgow Coma Scale

*Student $t$ test

tMann-Whitney $U$ test

$\neq \chi 2$ test

Table 2 In-hospital Treatment of LHI patients with favorable and unfavorable outcome

\begin{tabular}{|c|c|c|c|}
\hline & Unfavorable (mRS score 4-6) $(n=140)$ & Favorable (mRS score $0-3)(n=113)$ & $P$ value \\
\hline \multicolumn{4}{|l|}{ In-hospital Treatments, n(\%) } \\
\hline Thrombolysis $^{a}$ & $5(3.6)$ & $2(1.8)$ & 0.466 \\
\hline Decompressive surgery $^{a}$ & $22(15.7)$ & $2(1.8)$ & $<0.001$ \\
\hline Mechanical ventilation ${ }^{a}$ & $28(20.00)$ & $2(1.8)$ & $<0.001$ \\
\hline Osmotic agents ${ }^{\mathrm{a}}$ & $125(89.3)$ & 79(69.9) & $<0.001$ \\
\hline Statins in acute phase ${ }^{a}$ & $36(25.7)$ & $53(46.9)$ & $<0.001$ \\
\hline Antiplatelets ${ }^{a}$ & $80(57.1)$ & 106(93.8) & $<0.001$ \\
\hline Anticoagulants ${ }^{\mathrm{b}}$ & $6(4.3)$ & $12(10.6)$ & 0.083 \\
\hline Antihypertensives $^{\mathrm{b}}$ & $35(25.0)$ & $25(22.1)$ & 0.657 \\
\hline Antidiabetic drugs ${ }^{\mathrm{b}}$ & 19(13.6) & $16(14.2)$ & 0.893 \\
\hline
\end{tabular}

${ }^{a}$ Acute phase treatment

${ }^{b}$ Percentage is calculated for patients with indication of the treatment for secondary prevention 
Table 3 Stroke-related complication during hospitalization of LHI patients with favorable and unfavorable outcome

\begin{tabular}{|c|c|c|c|}
\hline & Unfavorable (mRS score 4-6) $(n=140)$ & Favorable (mRS score $0-3)(n=113)$ & $P$ value \\
\hline Stroke-related complications, n(\%) & 128(91.4) & $63(55.8)$ & $<0.001$ \\
\hline \multicolumn{4}{|l|}{ Neurological complications, n(\%) } \\
\hline Brain edema & 75(53.6) & $5(4.4)$ & $<0.001$ \\
\hline Hemorrhagic transformation & $46(32.9)$ & $25(22.1)$ & 0.068 \\
\hline Seizures/epilepsy & $11(7.9)$ & $7(6.2)$ & 0.634 \\
\hline Central hyperthermia & $9(6.4)$ & $2(1.8)$ & 0.118 \\
\hline Recurrent stroke & $3(2.1)$ & $0(0)$ & $0.256^{*}$ \\
\hline \multicolumn{4}{|l|}{ Medical complications, n(\%) } \\
\hline Pneumonia & $94(67.1)$ & $41(36.3)$ & $<0.001$ \\
\hline Urinary tract infection & $14(10.0)$ & $5(4.4)$ & 0.148 \\
\hline Gastrointestinal bleeding & 25(17.9) & $4(3.5)$ & 0.001 \\
\hline Electrolyte disturbance & $56(40.0)$ & $22(19.5)$ & $<0.001$ \\
\hline Acute renal failure & 18(12.9) & $0(0)$ & $<0.001^{*}$ \\
\hline Urinary incontinence & $39(27.9)$ & $8(7.1)$ & $<0.001$ \\
\hline Bedsore & $11(7.9)$ & $2(1.8)$ & 0.042 \\
\hline Deep venous thrombosis & $6(4.3)$ & $3(2.7)$ & 0.735 \\
\hline Falls & $3(2.1)$ & $0(0)$ & $0.256^{*}$ \\
\hline
\end{tabular}

"Fisher exact test

the mMCAi [14]. Thus, we conducted a prospective, non-selective cohort of patients with LHI to elucidate the factors that directly correlate with favorable outcome at 90 days. In the present study, we found that more than one third of patients with LHI have relatively favorable clinical outcomes at 90 days. In the multivariable logistic regression analyses, adjusted for potential confounding factors, we found that (1) younger age, lower baseline NIHSS score and statins used in the acute phase were independently related to favorable outcomes; (2) It was maligant brain edema and increased risk of pneumonia concommitant with surgery, rather than decompressive surgery itself independently associated with unfavorable outcome.

Table 4 Factors associated with 3-month favorable outcome in LHI patients

\begin{tabular}{|c|c|c|c|}
\hline Varibles & Univariate analysis & Multivariate analysis (model 1$)^{*}$ & Multivariate analysis (model 2$)^{*}$ \\
\hline Age & $0.95(0.93-0.97)$ & $0.95(0.92-0.97)$ & $0.95(0.92-0.98)$ \\
\hline Baseline NIHSS score & $0.86(0.82-0.90)$ & $0.87(0.82-0.92)$ & $0.90(0.84-0.96)$ \\
\hline Hypertension & $0.44(0.26-0.73)$ & $0.38(0.18-0.78)$ & \\
\hline Baseline serum glucose & $0.90(0.82-0.98)$ & & \\
\hline Decompressive surgery & $0.10(0.02-0.42)$ & $0.11(0.01-0.92)$ & \\
\hline Ventilatory Support & $0.07(0.02-0.31)$ & & \\
\hline Antiplatelets & $5.24(2.23-12.31)$ & & \\
\hline Statins & $2.55(1.50-4.33)$ & $2.75(1.35-5.59)$ & $2.49(1.10-5.65)$ \\
\hline Pneumonia & $0.28(0.17-0.47)$ & & $0.42(0.19-0.93)$ \\
\hline Gastrointestinal bleeding & $0.17(0.06-0.50)$ & & \\
\hline Electrolyte disturbance & $0.36(0.20-0.65)$ & & \\
\hline Bedsore & $0.21(0.05-0.97)$ & & \\
\hline Urinary incontinence & $0.20(0.09-0.44)$ & & \\
\hline Brain edema & $0.04(0.02-0.10)$ & & $0.05(0.01-0.21)$ \\
\hline
\end{tabular}

Variables which had a significant association with favorable outcome in univariate analysis were listed $(P<0.05)$. Figures in parentheses are $95 \%$ confidence intervals $(\mathrm{Cl})$

Model 1: adjusted for variables with $p<0.05$ on univariate analyses excluding stroke-related complication

Model 2: adjusted for variables with $p<0.05$ on univariate analyses including stroke-related complication

*Adjusted odds ratios (OR) with $p<0.05$ in the multivariate logistic regression analysis 


\section{Age}

Whereas age is the leading risk factor and the strongest predictor of clinical outcome after ischemic stroke [31, 32], the influence of age on outcome has not yet been well investigated in LHI. As a general rule, young and middle-aged patients have less compensation capacity for space-occupying intracranial lesions than older patients with cerebral atrophy, however, those patients tend to have fewer comorbid conditions that are likely to increase the risk of mortality and poor functional outcome [33]. The result of our study is in line with previous studies conducted in LHI patients that received DHC [23-25], which reported that younger age was associated with favorable outcome in non-selective patients with LHI.

\section{Statins in acute phase}

Of note, in our cohort, among those patients with favorable outcome at 90 days, $46.9 \%(53 / 113)$ use statins in acute phase of stroke, but only $25.7 \%(36 / 140)$ of those without favorable outcome use statins. Results from multivariable analysis of our cohort showed that statins use in acute phase was an independent predictor for 3-month favorable outcome in patients with LHI, regardless of the models adjusting for potential confounders by including or excluding stroke-related complications. Statins have been widely used for primary and secondary prevention of stroke for many years. Recently, statins have been found to play an important role in the neuroprotection of acute ischemic stroke in animal models, due to its pleiotropic effects besides lipid-lowering such as vasodilatory, angiogenesis, neurogenesis, synaptogenesis, antithrombotic, anti-inflammatory, antioxidant and anticonvulsant effects [34-36]. Meanwhile, cerebral arterial occlusion has been associated with reduced infarct volume and improved neurological function with use of statins in experimental animal models [34-36]. A recent research showed that pretreatment with statins is associated with better outcomes regarding neurological improvement, disability, survival, and stroke recurrence in large artery atherosclerotic stroke [37]. A meta-analysis including 113,148 stroke patients suggested an association between prestroke statins use and improved stroke outcome at 90 days [38]. Based on available evidence, it is recommended to continue with prestroke statin treatment in the acute phase [21]; however, routinely prescribing statins as neuroprotective agents is still controversial. Moreover, there are limited available data evaluating the association between statins use in acute phase and favorable outcome in patients with LHI. The result of our study provides initial evidence that statins used in acute phase may be associated with favorable outcome in patients with LHI. Considering the limitations of observational study, randomized controlled trials are needed to validate the findings.

\section{DHC and stroke-related complications}

In the present study, we found that patients with favorable outcome less frequently had received DHC and mechanical ventilation than those without favorable outcomes. Multivariable analysis not including stroke-related complications showed that DHC was independently associated with unfavorable outcome (model 1). However, when stroke-related complication was taken into consideration, DHC was no longer an independent factor correlated with unfavorable outcome, while brain edema and pneumonia were independently associated with unfavorable outcome (model 2). Data from a previous pooled analysis of three randomized trials had demonstrated that DHC reduced mortality without increasing the risk of very severe disability among patients 60 years of age or younger with mMCAi [6]. DESTINY II trial indicated that DHC increased survival among patients older than 60 years of age with mMCAi, but most survivors were left with disabilities and needed assistance for daily living [39], which was also confirmed in a small sample randomized trial with Chinese patients [40]. The results of present study showed that DHC was no longer independent factor associated with unfavorable outcome in patients with LHI after adjusting for age, baseline NIHSS score and stroke-related complication. Therefore, we could reasonablely speculate that it was malignant brain edema and increased risk of pneumonia concomitant with surgery, rather than DHC itself were independently associated with unfavorable outcome. In-hospital medical complications due to DHC can impact on the clinical outcomes of the patient with LHI. A national inpatient sample database over a 6-year period in the United States found the rates of pneumonia to be $11.1 \%$ in 252 patients studied and was associated with an increased rate of mortality [41]. In a cohort study with patients undergoing DHC for space-occupying LHI, $42.4 \%$ of the patients suffered from at least one postoperative complication, with pulmonary problems being the most common cause (39\% of all complications) [17]. Stroke-related pneumonia was the most common medical complication in our study (36.3\% in patients with favorable outcomes and $67.1 \%$ in those with unfavorable outcomes) and patients complicated with pneumonia showed $42 \%$ (95\% CI 0.19-0.93) chance of favorable outcome compared with those patients without, after adjusting for age, baseline NIHSS score and other confounders. Since most pneumonia are potentially preventable or treatable, doctors should pay rigorous attention to the prevention, early detection and treatment of stroke-related pneumonia because of the higher events risk and concomitant poor outcome.

\section{Limitations}

The results of the present study should be interpreted with caution given its limitations. First, it was a single hospital-based study, with limited generalisability. Some patients with LHI might not be hospitalized, especially 
those who died before admitted to hospital, so we could not exclude inclusion bias in this study. Second, we only conduct a 3-month follow-up so that the predictive effect on long-term outcomes remains unclear. Therefore, we cannot advise whether the factors we identified associated with favorable outcome in patients with LHI also have a long-term effect. Third, since our hospital is one of national advanced stroke center in China, admission to a stroke unit, early mobilisation, bedside swallow evaluation and rehabilitation have become routine practice in even every stroke patients, our prognostic model of LHI did not incorporate these factors such as admission to a stroke unit, early mobilisation, swallow status or other life-threatening impairment. Finally, follow-up in our study was performed by telephone interview or postal questionnaire instead of a clinic visit which may result in a reporting bias.

\section{Conclusions}

We identified that more than one third of patients with LHI have relatively favorable clinical outcomes at 90 days.Younger age, lower baseline NIHSS score, absence of brain edema and pneumonia and statins used in the acute phase may be directly related to favorable outcome in patients with LHI. The result of our study provides preliminary evidence that the use of statins in the acute phase was associated with favorable outcome in patients with LHI. Future studies are needed to verify the findings of current study.

\section{Abbreviations}

95\%Cl: 95\% confidence interval; CT: Computed tomography;

DHC: Decompressive hemicraniectomy; LHI: Large hemispheric infarction; MCA: Middle cerebral artery; mMCAi: Malignant middle cerebral artery infarction; MRI: Magnetic resonance imaging; mRS: Modified Rankin Scale: NIHSS: National Institutes of Health Stroke Scale; OR: Odds ratio

\section{Funding}

This research was funded by Key Research and Development Program, Science and Technology Department of Sichuan Province in China (Grant NO.2017SZ0007)

\section{Availability of data and materials}

All relevant data are included in the manuscript. The individual data collected for each participant in our study cannot be provided in order to protect the participants' identity.

\section{Authors' contributions}

$J$, PZ - collected, analyzed and interpreted the data, as well as drafted the manuscript. SW, XY - participated in study conception and design, data interpretation and revised the manuscript. CW, ML - contributed substantially to study design and supervision, data interpretation and manuscript writing. All authors critically revised the manuscript for important intellectual content and approved the final manuscript.

\section{Ethics approval and consent to participate}

The study protocol was submitted to and approved by the Ethics Committee of People's Hospital of Deyang City.Written informed consent was obtained from all patients before they were enrolled, or from their legal proxies if the patient lost capacity to give the consent.

Consent for publication

Not applicable.

\section{Competing interests}

The authors declare that they have no competing interests.

\section{Publisher's Note}

Springer Nature remains neutral with regard to jurisdictional claims in published maps and institutional affiliations.

Received: 19 June 2018 Accepted: 7 September 2018

Published online: 20 September 2018

\section{References}

1. Huttner HB, Schwab S. Malignant middle cerebral artery infarction: clinical characteristics, treatment strategies, and future perspectives[J]. The Lancet Neurology. 2009;8(10):949-58.

2. Schwab S, Aschoff A, Spranger M, et al. The value of intracranial pressure monitoring in acute hemispheric stroke[J]. Neurology. 1996;47(2):393-8.

3. Steiner $T$, Ringleb $P$, Hacke $W$. Treatment options for large hemispheric stroke[J]. Neurology. 2001;57(suppl 2):S61-8.

4. Bardutzky J, Schwab S. Antiedema therapy in ischemic stroke[J]. Stroke. 2007:38(11):3084-94.

5. Heiss WD. Malignant MCA infarction: pathophysiology and imaging for early diagnosis and management decisions[J]. Cerebrovasc Dis. 2016:41(1-2):1-7.

6. Vahedi $\mathrm{K}$, Hofmeijer J, Juettler $\mathrm{E}$, et al. Early decompressive surgery in malignant infarction of the middle cerebral artery: a pooled analysis of three randomised controlled trials[]]. The Lancet Neurology. 2007;6(3):215-22.

7. Rahme R, Curry $R$, Kleindorfer $D$, et al. How often are patients with ischemic stroke eligible for decompressive hemicraniectomy?[J]. Stroke. 2012;43(2): 550-2.

8. Frank Jl. Large hemispheric infarction, deterioration, and intracranial pressure[J]. Neurology. 1995;45(7):1286-90.

9. Krieger DW, Demchuk AM, Kasner SE, et al. Early clinical and radiological predictors of fatal brain swelling in ischemic stroke[J]. Stroke. 1999;30(2): 287-92

10. Kasner SE, Demchuk AM, Berrouschot J, et al. Predictors of fatal brain edema in massive hemispheric ischemic stroke[]]. Stroke. 2001;32(9):2117-23.

11. Oppenheim C, Samson $Y$, Manai $R$, et al. Prediction of malignant middle cerebral artery infarction by diffusion-weighted imaging[J]. Stroke. 2000; 31(9):2175-81.

12. Thomalla GJ, Kucinski $T$, Schoder $\mathrm{V}$, et al. Prediction of malignant middle cerebral artery infarction by early perfusion-and diffusion-weighted magnetic resonance imaging[J]. Stroke. 2003;34(8):1892-9.

13. Hofmeijer J, Algra A, Kappelle $\sqcup$, et al. Predictors of life-threatening brain edema in middle cerebral artery infarction[J]. Cerebrovasc Dis. 2008;25(1-2):176-84.

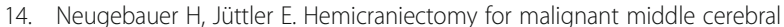
artery infarction: current status and future directions[J]. Int J Stroke. 2014; 9(4):460-467.

15. Qureshi Al, Suarez Jl, Yahia AM, et al. Timing of neurologic deterioration in massive middle cerebral artery infarction: a multicenter review[J]. Crit Care Med. 2003;31(1):272-7

16. Yi $X$, Lin J, Wang $C$, et al. Low-molecular-weight heparin is more effective than aspirin in preventing early neurologic deterioration and improving sixmonth outcome[J]. J Stroke Cerebrovasc Dis. 2014;23(6):1537-44.

17. Uhl E, Kreth FW, Elias B, et al. Outcome and prognostic factors of hemicraniectomy for space occupying cerebral infarction[J]. journal of neurology. Neurosurgery \& Psychiatry. 2004;75(2):270-4.

18. De Haan $R$, Limburg M, Bossuyt $P$, et al. The clinical meaning of Rankin 'handicap'grades after stroke[J]. Stroke. 1995;26(11):2027-30.

19. Adams HP, Bendixen BH, Kappelle $L$, et al. Classification of subtype of acute ischemic stroke. Definitions for use in a multicenter clinical trial. TOAST. Trial of org 10172 in acute stroke treatment[J]. Stroke. 1993;24(1):35-41.

20. Kernan WN, Ovbiagele B, Black HR, et al. Guidelines for the prevention of stroke in patients with stroke and transient ischemic attack: a guideline for healthcare professionals from the American Heart Association/American Stroke Association[J]. Stroke. 2014;45(7):2160-236.

21. Jauch EC, Saver JL, Adams HP, et al. Guidelines for the early management of patients with acute ischemic stroke: a guideline for healthcare professionals from the American Heart Association/American Stroke Association[J]. Stroke. 2013:44(3):870-947.

22. Johnston KC, Li JY, Lyden PD, et al. Medical and neurological complications of ischemic stroke: experience from the RANTTAS trial[J]. Stroke. 1998;29(2): 447-53. 
23. Schwab S, Steiner T, Aschoff A, et al. Early hemicraniectomy in patients with complete middle cerebral artery infarction[J]. Stroke. 1998;29(9):1888-93.

24. Mori K, Nakao Y, Yamamoto T, et al. Early external decompressive craniectomy with duroplasty improves functional recovery in patients with massive hemispheric embolic infarction: timing and indication of decompressive surgery for malignant cerebral infarction[]]. World Neurosurgery. 2004;62(5):420-9.

25. Gupta R, Connolly ES, Mayer $\mathrm{S}$, et al. Hemicraniectomy for massive middle cerebral artery territory infarction: a systematic review[]]. Stroke. 2004;35(2): 539-43.

26. Pillai A, Menon SK, Kumar S, et al. Decompressive hemicraniectomy in malignant middle cerebral artery infarction: an analysis of long-term outcome and factors in patient selection[J]. J Neurosurg. 2007;106(1):59-65.

27. Wang KW, Chang WN, Ho JT, et al. Factors predictive of fatality in massive middle cerebral artery territory infarction and clinical experience of decompressive hemicraniectomy[J]. Eur J Neurol. 2006;13(7):765-71.

28. Maramattom BV, Bahn MM, Wijdicks EFM. Which patient fares worse after early deterioration due to swelling from hemispheric stroke?[]]. Neurology. 2004;63(11):2142-5.

29. Rabinstein AA, Mueller-Kronast N, Maramattom BV, et al. Factors predicting prognosis after decompressive hemicraniectomy for hemispheric infarction[]]. Neurology. 2006;67(5):891-3.

30. Wartenberg KE. Malignant middle cerebral artery infarction[]]. Curr Opin Crit Care. 2012;18(2):152-63.

31. Nakayama $\mathrm{H}$, Jørgensen $\mathrm{HS}$, Raaschou $\mathrm{HO}$, et al. The influence of age on stroke outcome. The Copenhagen stroke study[]]. Stroke. 1994;25(4):808-13.

32. Macciocchi SN, Diamond PT, Alves WM, et al. Ischemic stroke: relation of age, lesion location, and initial neurologic deficit to functional outcome[J]. Arch Phys Med Rehabil. 1998;79(10):1255-7.

33. Schwab S. Therapy of severe ischemic stroke: breaking the conventional thinking[J]. Cerebrovasc Dis. 2005;20(Suppl. 2):169-78.

34. Vaughan CJ, Delanty N. Neuroprotective properties of statins in cerebral ischemia and stroke[J]. Stroke. 1999;30(9):1969-73.

35. Chen J, Zhang ZG, Li Y, et al. Statins induce angiogenesis, neurogenesis, and synaptogenesis after stroke[J]. Ann Neurol. 2003;53(6):743-51.

36. Di Napoli P, Taccardi AA, Oliver M, et al. Statins and stroke: evidence for cholesterol-independent effects[J]. Eur Heart J. 2002;23(24):1908-21.

37. Tsivgoulis G, Katsanos AH, Sharma VK, et al. Statin pretreatment is associated with better outcomes in large artery atherosclerotic stroke[J]. Neurology. 2016;86(12):1103-11.

38. Chróinín DN, Asplund K, Åsberg S, et al. Statin therapy and outcome after ischemic stroke[J]. Stroke. 2013:44(2):448-5.

39. Jüttler $E$, Unterberg A, Woitzik J, et al. Hemicraniectomy in older patients with extensive middle-cerebral-artery stroke[J]. N Engl J Med. 2014;370(12): 1091-100

40. Zhao J, Su YY, Zhang Y, et al. Decompressive hemicraniectomy in malignant middle cerebral artery infarct: a randomized controlled trial enrolling patients up to 80 years old[J]. Neurocrit Care. 2012;17(2):161-71.

41. Alshekhlee A, Horn C, Jung R, et al. In-hospital mortality in acute ischemic stroke treated with hemicraniectomy in US hospitals[J]. J Stroke Cerebrovasc Dis. 2011;20(3):196-201.

\section{Ready to submit your research? Choose BMC and benefit from:}

- fast, convenient online submission

- thorough peer review by experienced researchers in your field

- rapid publication on acceptance

- support for research data, including large and complex data types

- gold Open Access which fosters wider collaboration and increased citations

- maximum visibility for your research: over $100 \mathrm{M}$ website views per year

At $\mathrm{BMC}$, research is always in progress.

Learn more biomedcentral.com/submissions 